\title{
Community Opioid Overdose Prevention and Naloxone Distribution Programs: An Evidence-to-Practice Review
}

Madison M. Hauge, SCAT, ATC; Kathryn C. Downs, SCAT, ATC; Zachary K. Winkelmann, PhD, SCAT, ATC

University of South Carolina, Columbia, SC

\begin{abstract}
According to the Centers for Disease Control and Prevention, 100 people die of drug overdose in the United States every day. This frighteningly high mortality rate has created the need for community-based opioid overdose prevention programs (OOPPs). Currently, there are more than 188 community-run programs operating in the United States. These programs teach individuals how to distribute naloxone and respond properly to a drug overdose situation. This guiding systematic review depicts the current literature available on OOPPs and their effectiveness. The authors performed an article search to discover the most relevant and recent articles, which were graded using a quality assessment score. The search uncovered 19 articles deemed appropriate to investigate the effectiveness of OOPPs. Out of the 19 articles, 14 of these articles were cohort studies with large sample sizes that did a baseline and follow-up survey at two different time periods. Almost $50 \%$ of participants in this review stated they personally experienced an opioid overdose in their life. Furthermore, there was $79.2 \%$ of participants (across the 8 studies that report this data) to witness a drug overdose. Nonmedical bystanders is defined as individuals who could properly reverse an opioid overdose when OOPP training was completed. Eleven studies detected a $100 \%$ survival rate post-naloxone administration with the remaining 8 studies not far behind with a survival rate range of $83-96 \%$. The current evidence available suggests that OOPPs are successful in teaching their participants how to properly treat an opioid overdose with the administration of naloxone.
\end{abstract}

Key Phrases

Drug overdose/drug therapy, program evaluation, opioid-related disorders/drug therapy, naloxone/therapeutic use

\section{Correspondence}

Dr. Zachary Winkelmann, 1300 Wheat Street, Columbia, SC 29208.

E-mail:winkelz@mailbox.sc.edu

Twitter: @zachwinkelmann

\section{Full Citation}

Hauge MM, Downs KC, Winkelmann ZK. Community Opioid Overdose Prevention and Naloxone Distribution Programs: An Evidence-to-Practice Review. Clin Pract Athl Train. 2021;4(2): 33-39. https://doi.org/10.31622/2021/0004.2.5.

Submitted: April 20, 2020 Accepted: November 16, 2021.

\section{ORIGINAL REFERENCE}

Clark AK, Wilder CM, Winstanley EL. A systematic review of community opioid overdose prevention and naloxone distribution programs. J Addict Med. 201 4;8(3):153-163.

\section{SUMMARY}

\section{CLINICAL PROBLEM AND QUESTION}

\section{According to the World Health Organization,}

the three main symptoms for an opioid overdose (generally termed "opioid overdose triad") are pinpoint pupils, unconsciousness, and respiratory depression. ${ }^{1}$ This fatal combination can ultimately result in death. In fact, 128 people die every day in the United States from opioid overdose. ${ }^{2,3}$ According to the Centers for Disease Control and Prevention, opioid overdose is a rising issue in the United States with a $9.6 \%$ increase in the ageadjusted rate in overdose deaths from 2016 to 2017.4 In 2017, there were a total of 70,237 drug overdose deaths. ${ }^{2}$

To combat this public health emergency, opioid overdose prevention programs (OOPPs) were created to educate the public about how to recognize and manage an opioid overdose with treatment training using naloxone.2,5 Naloxone, which is commonly referred to by its brand name Narcan, is an opioid antagonist used to temporarily reverse a drug overdoses in an emergency situation. However, the drug is relatively new, and the opioid issue has continued to grow. In addition, OOPPs cover the risk factors of opioid overdose, as well as, the procedure of how to properly respond to an overdose with or without naloxone.1,2,4 Opioid overdose prevention 
programs are essential since they advocate for prevention efforts such as encouraging people (e.g., people at risk, the family members of people considered 'at risk', and healthcare providers) to learn how to manage a drug overdose.

Opioid overdose prevention programs have been utilized in the United States for 24 years; though, there is very limited research on the programs' impact and outcomes of the training. ${ }^{5-7}$ This evidence-to-practice review summarizes a systematic review to analyze the impact of OOPPs by evaluating if naloxone distribution reduces the instances of overdose among their participants. ${ }^{3}$ This review also compared the United States OOPPs effectiveness on increasing bystander knowledge on prevention, risk factors, and detection of and response to an opioid overdose. ${ }^{3}$ Lastly, it appraises how OOPPs participants respond to opioid overdose. Overall, this systematic review organizes the available current literature on opioid overdose to observe the general impact of OOPPs.

\section{SUMMARY OF LITERATURE}

The guiding systematic review searched databases including PubMed, MEDLINE, and PsychINFO, using the Boolean search query: (opioid OR opiate) AND overdose AND prevention. The search was limited to English language, which returned 360 different citations. The citations were sorted and included only original, peerreviewed articles examining community OOPPs that detected a training impact. This consisted of reports of overdose reversal rates, overdose fatalities, or any measure of overdose rate among program participants. Exclusion criteria included OOPPs that did not incorporate training on the use or distribute naloxone, data that was unable to separate into program-specific information, and program evaluations that focused more on health care personnel training. Thirty-eight articles were identified for full-text review; however, 19

Copyright $(\subset)$ by Indiana State University All rights reserved. ISSN Online 2577-8188 articles fit the exact inclusion conditions and were included in this review. The remaining 17 were excluded because these articles did not assess a community OOPP (11), did not report a training outcome (2), did not have naloxone training (1), or were based on grouped or clumped data (2). A quality appraisal was completed on the articles involved in this systematic review. The quality score had a possible range of $4-8$ and this set of studies used in the review had a mean of 6.1, median of 6.5, and a mode of 7. Although majority of these studies scored a 0 for randomization and low rates for follow-up, $18 / 19$ of these studies got a maximum score for sample size and outcomes. Overall, the studies were graded as fair based off descriptive quality.

\section{SUMMARY OF INTERVENTION}

The intervention investigated in the systematic review were OOPPs. The systematic review focused on the effectiveness of the OOPPs and their outcomes within the respective communities. The OOPP intervention ranged in duration from 10-60 minutes. The laws for prescribing naloxone varied by state, as well as, the physician involvement with the OOPPs. Because of this, the qualifications of the personnel instructing the interventions differed between programs and in most studies the qualifications of the instructors were not specified. Naloxone prescription is variable between states so involvement with physicians in OOPPs ranged from notification of program completion to meeting with a physician in order to receive a naloxone kit. ${ }^{8-10}$

A majority of the programs include a curricula based on five components including: 1) recognizing overdose $(78.9 \%)$, 2) preventing overdose $(73 \%), 3)$ risk factors of overdose $(63 \%), 4)$ appropriate response to overdose $(84 \%)$ and, 5) administration of naloxone (100\%) with some variation and deviations in the material provided. The response to overdose, such as 
rescue breathing, cardiopulmonary resuscitation $(C P R)$, and the recovery position (Figure 1), were addressed in the majority of OOPPs. In addition, administering naloxone in a variety of ways and at varying levels of practice was a part of the curricula in each of the intervention programs. Fifteen of the articles provided training with needle-based administration, however, some programs provided training practice by injecting into an orange and while other programs used nasal naloxone. 11-13

\section{SUMMARY OF OUTCOMES}

The guiding systematic review analyzed outcomes of the OOPPs. The first analysis was for the outcomes associated with the reduction of fatal and nonfatal overdose rates for those who participated in an OOPP intervention. Naloxone was used successfully 1,949 times by those who attended OOPPs. This review identified heroin as the most frequently reported overdosed drug, despite the National Vital Statistics System indicating opioid analgesics as the highest drug used. The studies reported that the survival postnaloxone administration rate ranged from $83 \%$ $100 \%$ with 11 of the studies reporting a $100 \%$ survival rate. Two of the articles that reported lower survival rates attribute the findings to unknown overdose outcomes. ${ }^{10,14}$ In addition, two studies found that areas with higher OOPP enrollment demonstrated decreased opioid overdose mortality at a population level. 13,15 With the majority of studies demonstrating $100 \%$ survival after administration of naloxone by those who attended OOPPs, we conclude that OOPPs have the ability to reduce fatal and nonfatal overdoses among participants.

Next, the review analyzed the outcomes on the effectiveness of OOPPs to increase nonmedical bystander knowledge of prevention, risk factors and recognition of opioid overdose and correct response. Just under half of the studies in the review reported pre- and post-training measures

Copyright $(\subset)$ by Indiana State University All rights reserved. ISSN Online 2577-8188

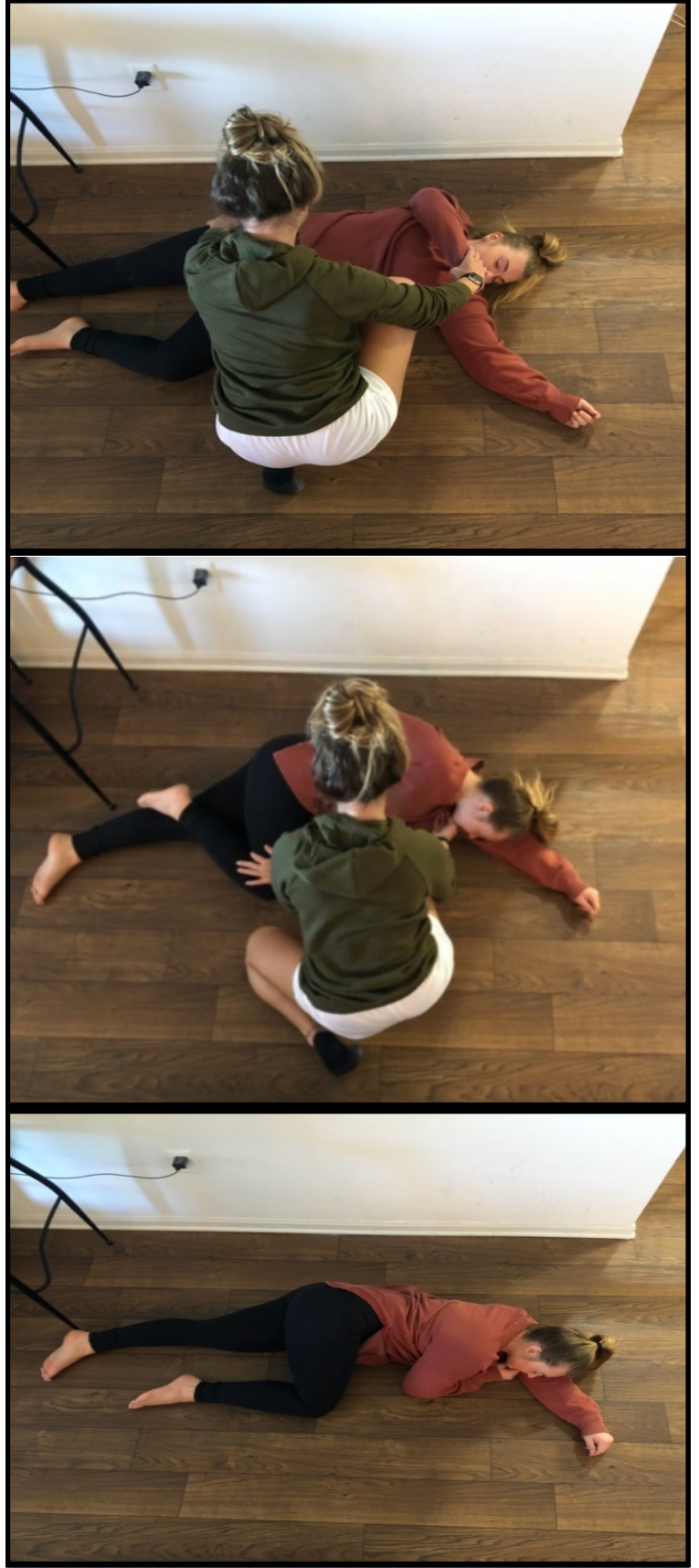

Figure 1. Demonstration of the recovery position in drug overdose management

regarding the knowledge surrounding opioid overdose. The consensus among these studies is that those who participated in OOPPs had an increase in their knowledge of prevention, risk factors, and recognition of opioid overdose. 16-21 
In terms of response, participants in OOPPs displayed a behavior change with an increase in appropriate responses like rescue breathing, administering naloxone, initiating recovery position, and performing a sternal rub. 10,14, 17, 22 However, in two studies there were reports of nonrecommended responses, such as using ice and cold water to revive patients. ${ }^{21,} 22$ These results display that the curriculum in studies that utilized pre- and post-training measures increased the knowledge of nonmedical bystanders.. This finding can be interpreted to show that nonmedical personnel have the ability to learn prevention, risk factors, and recognition of opioid overdose and therefore expand the population of those who can respond to opioid overdoses.

When considering the following outcomes associated with the reduction of fatal and nonfatal overdose rates for those who participated in an OOPP intervention and effectiveness of OOPPs to increase nonmedical bystander knowledge of prevention, risk factors and recognition of opioid overdose and correct response the literature shows that OOPPs may have the ability to create meaningful change within communities. OOPPs can reduce fatal and non-fatal overdoses. The data suggests the ability for non-medical bystanders to learn about and properly respond to opioid overdoses. However, due to the nature of the studies and the way they have been conducted these, conclusions are limited due to the lack of randomization.

\section{FINDINGS AND CLINICAL IMPLICATIONS}

Due to the lack of randomization and systematic measures, it is difficult to create generalizable statements and conclusions of the effectiveness of the programs when the specific content is not documented. Although the evidence is not concrete, we can deduce that OOPPs have the potential to reduce opioid overdose morbidity and mortality. Clinically, to reproduce similar outcomes within our communities, athletic trainers

Copyright $\left({ }^{\circ}\right.$ by Indiana State University All rights reserved. ISSN Online 2577-8188 and non-medical bystanders need to be educated on the five components of OOPPs: recognizing overdose, preventing overdose, risk factors for overdose, appropriate response to overdose, and administration of naloxone. We know that a $100 \%$ survival rate can be achieved by attending OOPPs, and communities should focus on administration of naloxone as responses to overdose such as rescue breathing and CPR.

Naloxone education and administration should be integrated into standards and practices of all first responder and healthcare providers. Standard 70 in the 2020 Commission on Accreditation of Athletic Training Education (CAATE) standards describe that professional athletic training programs must teach how to evaluate and manage patients with acute conditions, including triaging conditions that are life threatening or otherwise emergent including drug overdose. ${ }^{23}$ This includes the administration of naloxone. However, the goal should be to ensure that all ATs that completed formal education before this standard was implemented are also comfortable with the skill. This goal can also be achieved by incorporating elements of OOPPs into continuing education. By introducing OOPP education and training into athletic training continuing education we ensure athletic trainers can carry out the skills themselves as well as teach the skills in their communities. By educating athletic trainers we can use them as a bridge to educate their respective communities. Athletic trainers serve unique roles that allow for them to be very involved within their surrounding communities, especially those in the secondary school settings. Communities should take advantage of this role that athletic trainers hold and use them as a liaison to instruct OOPPs in the communities they serve. Studies show that non-medical bystanders have been deemed efficient and successful in administering naloxone to those who have overdosed. ${ }^{16-21}$ This means that athletic trainers can teach anyone the OOPP curriculum and they have the potential to be successful in carrying out those skills. Athletic 
trainers can be extremely valuable by encouraging the high-risk populations that they know and interact with to attend OOPPs and help to cultivate better outcomes within the community.

Moving forward, the development of a standardized OOPP could be used to effectively educate groups across the country about opioid overdose and naloxone administration. Before a standardized OOPP is created, there is an opportunity for an OOPP assessment tool to be used to validate current programs. This tool would work towards deeming OOPPs efficient in education covering the five components; recognizing overdose, preventing overdose, risk factors for overdose, appropriate response to overdose, and administration of naloxone. OOPP could be designed like first aid or CPR (cardiopulmonary resuscitation) certification programs that require vetted instructors to educate the participants on standardized information. No matter who teaches the course or where one takes the course, each participant will walk away with the same knowledge and abilities regarding opioid overdoses and naloxone administration. Again, this is where athletic trainers can come in and use the information and skills they have gained in their continuing education course to teach and carry out the OOPPs. The systematic review highlights a need for OOPPs. It explains that opioid overdose is common, and naloxone is efficient and safe in reversing opioid overdoses. Although these data are not methodologically sound, this guiding systematic review identifies a trend towards OOPPs having the potential to decrease morbidity and mortality with an opioid overdose.

\section{CLINICAL BOTTOM LINE}

Opioid overdose prevention programs have worked towards reversing opioid overdoses, but research has reported mixed findings for its overall effectiveness in reducing the number of opioid incidences. This current systematic review

Copyright $\left({ }^{\circ}\right.$ by Indiana State University All rights reserved. ISSN Online 2577-8188 was not able to determine this general effectiveness because of the lack of consistent measures and methodological limitations; however, the chief finding presented throughout the articles was that OOPPs were effective, specifically in the treatment of an opioid overdose and the administration of naloxone.

Evidence in this review detected that heroin was the most frequently reported drug before an overdose; however, the leading cause cited was opioids. ${ }^{24}$ This fact is essential to health care providers, since many injuries are prescribed with opioid analgesics to reduce injury pain. Because of this athletes are an at risk population for the misuse of these prescription opioids. Athletic trainers are suggested to monitor post-operative patients, or any patient given opioids, and checkin on their pain levels. The patient should not be reliant on opioids long term, so there should be a plan of action and timeline for gradually waning the patient off prescription pain relievers. The literature also did confirm that the majority of OOPP participants used non-recommended strategies, such as not call emergency medical services for an overdose. This is a factor that athletic trainers should emphasize in educating their patients, whether they believe medical assistance is needed or not, because there could be medical complications from the restricted respiration occurring in an overdose. ${ }^{23,24}$ The fear of police was indicated as the main reason for not preparing; however, the studies displayed more positive interactions than negative.16,17 Athletic trainers should direct attention to the prolonged medical issues an overdosed individual could have and attempt to ease the fear of utilizing EMS personnel. 21,22

It is key that athletic trainers, much like the OOPP, are able to prevent, recognize, and respond to opioid overdose using pharmacological and nonpharmacological interventions. We suggest that athletic trainers consider taking an OOPP, or a formal opioid overdose training course, if 
unfamiliar with the management techniques described in this review. Additionally, athletic trainers can play a role in preventive efforts by spreading opioid overdose information to their population, including signs and symptoms of overdose, red flags to look out for, how to recognize an overdose, and how to respond if they are the non-medical bystander in an overdose situation. If opioid overdose education was accessible, or even mandatory prior to sport participation, for athletes and parents, then such education could improve the awareness and discourage opioid abuse. With the knowledge on how to deal with an opioid overdose, athletes can offer life-saving assistance in responding to a family member's or roommates' overdose situation. An athletic trainer should distinguish how to respond with or without a naloxone kit present, so individuals are prepared for both scenarios. Overall, there is evidence that supports OOPPs may benefit how to effectively handle an overdose situation. However, research is needed on this subject with more of a standard instrument for measuring how well these programs assist their participants in managing a drug overdose.

\section{REFERENCES}

1. Information sheet on opioid overdose. (2018, August 21). Retrieved April 1, 2020, from https://www.who.int/substance abuse/infor mation-sheet/en/.

2. Hedegaard $H$, Miniño AM, Warner M. Drug overdose deaths in the United States, 19992017. NCHS Data Brief, no 329. Hyattsville, MD: National Center for Health Statistics. 2018.

3. Wheeler E, Jones TS, Gilbert MK, Davidson PJ. Opioid Overdose Prevention Programs Providing Naloxone to Laypersons - United States, 2014. MMWR Morb Mortal Wkly Rep. 2015;64(23):631-635.

4. Centers for Disease Control and Prevention. Vital signs: overdoses of prescription opioid pain relievers-United States, 1999-2008.

Copyright $(\subset)$ by Indiana State University All rights reserved. ISSN Online 2577-8188
Morb Mortal Wkly Rep 2011 ;60:14871492.

5. Scholl $L$, Seth $P$, Kariisa $M$, Wilson $N$, Baldwin G. Drug and Opioid-Involved Overdose Deaths - United States, 2013-2017. MMWR Morb Mortal Wkly Rep.2018;67(5152):1419-1427. DOI: $10.15585 / \mathrm{mmwr} . \mathrm{mm} 675152 \mathrm{el}$

6. Sporer KA, Kral AH. Prescription naloxone: a novel approach to heroin overdose prevention. Ann Emerg Med. 2007;49(2): 172-177. https://doi.org/10.1016/i.annemergmed.20 06.05.025.

7. NIDA. Opioid Overdose Crisis. National Institute on Drug Abuse website. https://www.drugabuse.gov/drugsabuse/opioids/opioid-overdose-crisis. February 20, 2020. Accessed April 1, 2020.

8. Yokell MA, Green TC, Bowman S, et al. Opioid overdose prevention and naloxone distribution in Rhode Island. Med Health Rhode Island. 2011 ;94:240-242.

9. Galea S, Worthington N, Markham Piper T, et al. Provision of naloxone to injection drug users as an overdose prevention strategy: early evidence from a pilot study in New York City. Addict Behav 2006;31:907-91 2. https://doi.org/10.1016/i.addbeh.2005.07 .020 .

10. Markham Piper T, Stancliff S, Rudenstine S, et al. Evaluation of a naloxone distribution and administration program in New York City. Subst Use Misuse 2008;43:858-870. https://doi.org/10.1080/1082608070180 $\underline{1261}$.

11. Bennett T, Holloway K. The impact of takehome naloxone distribution and training on opiate overdose knowledge and response: an evaluation of the THN Project in Wales. Drugs Educ Prev Policy 201 2; 19:320-328. https://doi.org/10.3109/09687637.2012. $\underline{658104}$.

12. Doe-Simkins $M$, Walley AY, Epstein A, et al. Saved by the nose: bystander-administered

Clinical Practice in Athletic Training Volume 4 - Issue 2 - September 2021 
intranasal naloxone hydrochloride for opioid overdose. Am J Public Health 2009;99:788791.

https://dx.doi.org/10.2105\%2FAJPH.2008. 146647.

13. Walley AY, Xuan $\mathrm{Z}$, Hackman $\mathrm{HH}$, et al. Opioid overdose rates and implementation of overdose education and nasal naloxone distribution in Massachusetts: interrupted time series analysis. BMJ 2013b;346:f174. https://doi.org/10.1136/bmi.f174.

14. Enteen L, Baver J, McLean R, et al. Overdose prevention and naloxone prescription for opioid users in San Francisco. J Urban Health 2010;87:931-941.

https://doi.org/10.1007/s1 1524-0109495-8.

15. Maxwell S, Bigg D, Stanczykiewicz K, et al. Prescribing naloxone to actively injecting heroin users: a program to reduce heroin overdose deaths. J Addict Dis 2006;25:8996.

https://doi.org/10.1300/j069v25n03 11.

16. Bennett T, Holloway K. The impact of takehome naloxone distribution and training on opiate overdose knowledge and response: an evaluation of the THN Project in Wales. Drugs Educ Prev Policy 2012; 19:320-328. https://doi.org/10.3109/09687637.2012. $\underline{658104 .}$

17. Tobin KE, Sherman SG, Beilenson $P$, et al. Evaluation of the Staying Alive programme: training injection drug users to properly administer naloxone and save lives. Int $J$ Drug Policy 2009;20:131-136. https://doi.org/10.1016/i.drugpo.2008.03. $\underline{002}$.

18. Strang J, Manning V, Mayet $S$, et al. Overdose training and take-home naloxone for opiate users: prospective cohort study of impact on knowledge and attitudes and subsequent management of overdoses. Addiction 2008a;103:1648-1657. https://doi.org/10.1111/i.13600443.2008.02314.x.
19. Lopez Gaston R, Best D, Manning V, et al. Can we prevent drug related deaths by training opioid users to recognise and manage overdoses? Harm Reduct J 2009;6:26. https://doi.org/10.1186/14777517-6-26.

20. McAuley A, Lindsey $G$, Woods $M$, et al. Responsible management and use of a personal take-home naloxone supply: a pilot project. Drugs Educ Prev Policy 2010;17:388-399. https://doi.org/10.3109/0968763080253 $\underline{0712 .}$

21. Sherman SG, Gann DS, Scott G, et al. A qualitative study of overdose responses among Chicago IDUs. Harm Reduct J 2008;5:2. https://doi.org/10.1186/14777517-5-2.

22. Lankenau SE, Wagner KD, Silva K, et al. Injection drug users trained by overdose prevention programs: responses to witnessed overdoses. J Community Health.

2013;38(1):133-141. https://doi.org/10.1007/s10900-0129591-7.

23. Standards for the Commission on Accreditation of Athletic Training Education Web site. https://caate.occutrain.net/wpcontent/uploads/2014/01/2012Professional-Standards.pdf. Accessed March 25, 2020.

24. Paulozzi LJ. Prescription drug overdoses: a review. J Saf Res 2012;43:283- 289. https://doi.org/10.1016/i.jsr.2012.08.009. 